https://doi.org/10.48009/2_iis_2006_59-63

\title{
SAP R/3 IMPLEMENTATION SUCCESS INCREASES AS ONE ORGANIZATION DEVIATES FROM FASTTRACK@ FOR SAP: A CASE STUDY
}

\author{
Dr. Gina Boff, California University of Pennsylvania, boff@cup.edu \\ Dr. Gary DeLorenzo, California University of Pennsylvania, delorenzo@cup.edu
}

\begin{abstract}
This study uncovered, through active participation in a four-year, ethnographic field research, how three ERP (Enterprise Resource Planning) projects became increasingly successful as each implementation evolved beyond the standard implementation methodology, with which it began, into a semi-customized approach.
\end{abstract}

Keywords: ERP, Enterprise Resource Planning, Implementation, SAP, FastTrack@

\section{INTRODUCTION}

"When the Gartner Group coined the term 'ERP' in 1990, it declared a new vision for resource planning within organizations" [1]. Enterprise Resource Planning (ERP) can offer a competitive edge to corporations by empowering highly diverse and/or global businesses to transform into streamlined business entities. With ERP enablement, companies once using disparate information systems can execute seamless business transactions using integrated information-technology solutions. Sixteen years after Gartner's vision, ERP enablement is still very much sought after, as indicated by a recent AMR Research study of mid-size to large companies, which states that " $67 \%$ of these companies are evaluating potential ERP systems solutions" with the overall ERP market expected to grow to $\$ 64.8$ billion by 2009 [8]. However, the means in achieving ERP enablement is not easy. In the March, 2006 Communications of the $A C M$, authors Beatty and Williams show that the cost of a typical ERP implementation in a Fortune 500 company is estimated between $\$ 40$ to $\$ 240$ million and achieving only $61 \%$ completion [3].

For Bayer AG, a multi-national chemical and health care corporation with 2005 annual sales of $\$ 35$ billion, these statistics did not stand. Through the course of a four-year study of three consecutive SAP $\mathrm{R} / 3$ implementations belonging to three, distinct businesses of Bayer $\mathrm{AG}$, this research uncovered ways in which these businesses undergoing ERP implementation initiatives became 'smarter' in the process over time and deviated from the popular, Deloitte \& Touche (D\&T) FastTrack@ for SAP [4] implementation methodology into something that better suited the Bayer AG businesses [5].

\section{LITERATURE REVIEW}

Different views on ERP success factors have been proposed in the literature, with just as many variables introduced as influences. Such variables include the corporate culture and social contracts in which the implementation is occurring $[9,10]$, team dynamics and the nature of task-related conflicts in the project implementation [4], project leadership, uppermanagement support, common time and cost constraints [5], complexity issues arising from legacy systems [7], incentive alignments with ERP roles, and even the "moral hazard" of consulting agencies" intentional misrepresentation of situational realities [2]. While a more thorough and lengthy explanation of this study exists for reference [5], the reader should find the scope of this paper narrow enough to be sensible in light of the brevity constraint.

\section{METHODOLOGY}

The foundation of this paper comes from first-hand experiences while actively participating in three successive implementations of SAP R/3. Table 1 shows the SAP R/3 projects used in the research, the status of the implementation during the study and the elapsed duration Bayer AG had spent on each implementation.

Table 1. SAP R/3 Projects

\begin{tabular}{|l|l|l|l|}
\hline ID & DESCRIPTION & STATUS & ELAPSED \\
\hline CC & Consumer Care & Past & 32 months \\
\hline PH & Pharmaceuticals & Present & 12 months \\
\hline AH & Animal Health & Future & 6 months \\
\hline
\end{tabular}

All three businesses used employed D\&T's FastTrack@ for SAP as their implementation methodology. [6]. Each implementation phased in all of the SAP modules and are considered, today, fully ERP enabled. These three, consecutive cases afforded a chronological perspective of the process and the ability to track any variations in the process over time. 
This study employed a multi-method qualitative approach in order to allow common themes to emerge and to dismiss single, chance occurrences as outliers or 'quirks'. In addition to active participation, the researchers used case study, ethnographic observation and documentation. Some of the sources used include, but are not limited to the following:

- A list of technological tools used by various team members to track various portions of the implementation. This includes things such as project-management tools, change-management tools, electronic project notebooks (EPN) and problem tracking software.

- Implementation methodology training scripts, manuals, books, process depictions and databases by subject.

- Organizational snapshots through time and implementation progress snapshots through time - status of project plans.

- Implementation project documents - these, all contained within various EPNs described, in painstaking detail, each and every business and technological step taken toward ERP enablement. These documents were an irreplaceable source of information.

- Meeting minutes and meeting agendas.

\section{Indexing, Validation and Categorical Aggregation}

A matrix was developed to identify pieces of information that could help in the discovery process. The matrix included the sources where the information might be obtained and the tools that would be employed to gain the information. Sources for gathering data included a review of corporate documents, observation and ethnographic interviews with key, selected team members.

This same matrix assisted in the categorization of the information. As data were collected, they were indexed according to their relevance to each indicator. Once the indexing was done, it was aggregated by category. This information coding process used Stake's aggregation of instances [11]. And finally, only those categories having the most entries were analyzed for further meaning and their implications to the study.

\section{Interpretation and Discovery}

The focus of this study was not necessarily the degree of success of the ERP system, but more on the success of the implementation of the ERP system.
While implementation success could have been defined in a variety of ways, the definition here was three-fold: (1) The time expended during the implementation process to deployment, (2) the number of implementation issues encountered prior to deployment and the speed and/or ease with which they were addressed and overcome, and (3) the degree of end-user satisfaction, in terms of clients using the system as designed and that they were satisfied with the results [2]. The reason why this was included as a measure is because the implementation could progress smoothly but if the end result does not solve the business problems for which it was developed, the implementation success is meaningless [2].

As users became more involved with processes better suited to their expertise, the time of each implementation decreased (see Table 1 - Elapsed), problems encountered during the implementation were less frequent and more efficiently addressed, and users seemed more satisfied with their ERP systems.

In addition, other patterns emerged that indicated a deviance from the steps taken in previous implementations. For example, concerns among team members were being addressed through informal means so as to bypass the formal process. Also, the teams influenced an evolution of existing policies and practices so that they could become more efficient as the ERP implementation processes moved through time. This led the ERP implementation process away from publicized practices to what worked for the particular environment. This surfaced the next point of the research, which was "What are the underlying deficiencies in the publicized ERP implementation methodologies that cause countless endeavors to fail and, often times, detrimentally effect the welfare of the corporation?" At this point, we backtracked through the data collected to compare the standard ERP implementation methodology (FastTrack(C), with which Bayer AG began, to what was actually occurring in the consecutive cases. These findings are discussed in the next section.

\section{RESULTS}

The results of the study stem from areas where Bayer AG diverged from the FastTrack@ implementation methodology and/or the points that were problematic at first but became easier as the company got "smarter" about its implementations. These deviations are restated here as "watch points" for companies wishing to take on the ERP 
implementation challenge. For a full explanation of the results, see Boff [3].

\section{Step One: Choose the ERP Technological Software Solution}

An extensive and time-consuming vendor selection is misplaced energy. ERP software vendors have progressed according to industry needs and demands. The key is to get a handle on at least one reengineered process prior to vendor selection. It is important to go into the selection process with a solid understanding on reengineered business processing. Limit the selection process to integrated solutions, focus on the system functionality, and develop a quick-pick procedure and stick to it. The success in ERP enablement is not achieved through believing in plug-and-play implementations; they do not exist.

\section{Step Two: Choose the Consultants}

The second step in the ERP implementation process lies in picking the consulting firm that help guide the project life cycle. There are four basic choices to the consulting component:

1. Choose one of the Big Five consulting firms.

2. Choose the consulting services offered by the largest technology-enabling vendors, such as IBM.

3. Choose the consulting services of the ERP software vendor.

4. Choose a smaller, independent ERP consulting firm.

The insights gained in the consulting selection process are to choose a consulting firm that has a culture which fits your own; choose from only those companies which are readily and logistically available to you; remember that technological integration is only a small piece of a big initiative; and compare track records on those consulting firms who have successfully ERP-enabled companies.

\section{Step Three: Begin BPR}

Business Process Reengineering (BPR) is the largest part of the ERP process. It is also the most difficult, confusing, and time-consuming part as well, but the real potential for reaping the benefits of ERP is directly related to how well BPR changes the current business. During this time, companies rely on the chosen consulting firm to lead the project efforts, by first teaching their implementation methodology to the BPR team. BPR learns the different roles in the project and how the segregation of project functions is used as a means of project management.
It is important to note that this is the only part of the process that can occur more than once in a given implementation. And if an initiative is disbanded, it is because of this BPR process. As the most difficult, the most time consuming and the most expensive part of ERP, it was an unsurpassed benefit to be able to watch this process barely complete the first time, and then to see the process evolve over time to become more efficient.

BPR Step A: Scoping and Planning. This stage involves defining and launching the ERP project, which is to involve a team of consultants and business staff to develop the business and technological objectives, scope, resource requirements, cost estimates, and timing of the project. It is during this phase that the business case is initiated and different "threads" are established to support the overall ERP initiative. These threads are made up of business groups who have expertise in various areas and will use this expertise to specialize in support functions necessary for project success. There are a few things that should continually remain in the project manager's radar:

1. Remain aware of where the corporation is, in reality, at this point in time.

2. Get the right people in the right places, from the start.

3. Introduce the BPR teams to deadlines and make them fully accountable for project success.

4. Start small and grow the BPR teams through the phases, rather than accounting for every possible skill that might be needed from the start.

It is important to keep in mind that ERP is driven by $B P R$. As such, it only makes sense that the threads are established to support BPR, which means that they are dependent upon the progress and success of $B P R$. BPR has a responsibility to the entire ERP initiative to give appropriate direction and detail to the supporting threads and to keep to their timelines. If the project falls behind, it is assuredly due to one or more of these missing.

BPR Step B: Visioning and Targeting. During this phase, the ERP team, now comprised of a team of consultants and teams of business experts and users, work to develop an enterprise vision that falls within the constraints of the ERP software. This vision is translated into a preliminary organizational model. Technological infrastructure requirements are developed from the model for the whole enterprise. Also during this phase, the project team is trained, and prototyping and simulation begin. Some of the pitfalls that Bayer AG fell into with the CC-ERP 
case, that were overcome in subsequent implementations, were as follows:

1. The more targeting and visioning, the more money goes to the consulting firm.

2. The process redesign must lead the software, and not vice versa.

3. Be realistic and focus on getting a system up even if it is not "the vision" - this must be the corporate goal, especially for the first deployment.

4. Be sure that those BPR team members assigned to business processes now have the expertise to move onto the next phase; otherwise, reassign as needed.

BPR Step C: Redesign. In this phase, the project team works with the business units to develop detailed designs for the business that fulfill the visions developed in the Visioning and Targeting phase. Software for data conversion, interfaces, custom forms and reports, and any required system enhancements are planned. The technology infrastructure required to execute the project is implemented and the approach for end-user training is designed.

This phase is very task-oriented. Still dependent on the previous phase of "Targeting and Visioning", the project teams fill in the gaps created by the "bluesky" design, created in the previous phase, with detail as to how the business processes are to function within the ERP system. If there was no governor applied to Targeting and Visioning, this phase becomes impossible.

In this phase, details are incorporated into the process. Processes are broken down into small steps and the elements necessary to execute each step are documented in what's known as "scripting". Each and every process that will exist in the ERP system is scripted, in addition to processes which lie outside of the ERP system but need to execute in order to complete a business process. These scripts serve as a basis on which to test the ERP system and to train future users. They also serve as the starting point by which information technology begins to gather requirements in order to create technical design specifications. Without the detail provided in the scripts, designing technical enhancements to fill these gaps would be more vulnerable. Be sure that the scripts solidify the details.

Another critical aspect of the project started here is the task of defining and loading master data. Unlike other tasks, the ERP system cannot function without master data and subsequent steps to the implementation can not effectively proceed without it. Be sure that enough up-front attention is given to master data. Other noteworthy activities are:

Reevaluate those BPR deadlines.

Continually evaluate the effectiveness of the BPR team members and their ability to drive though the tasks.

When BPR determines that something in the "blue sky" system is not possible, take immediate action.

Be realistic and focus on getting a system up, even if it is not "the vision".

BPR Step D: Configuration. In this phase, the project team configures SAP to support the detailed business design created in the redesign phase. Data conversion, interfaces and software enhancements are developed and tested with the configuration. A recommendation for transitioning the work force is presented and end-user training and development begin.

The task of configuration is spread throughout the entire process. Some configuration is needed in order to load master data; other configuration cannot be completed until master data is loaded, etc. The integration of configuration tasks with redesign cannot be stressed enough.

There are a number of insights gained that are noteworthy. These are as follows:

There must come a point in time where configuration is frozen.

Hold BPR to their deadlines or I.T. will get squeezed. Coordination between I.T. and BPR right now is paramount.

BPR Step E: Testing and Delivery. In this phase, the project team works with the business units to perform technical and business process integration tests. This ensures that the new business design is effectively incorporated into the new system. The organization is prepared for the upcoming transformation through knowledge transfer, end-user training and change leadership initiatives, which ensure a smooth transition to the new way of working in the ERP system. After the new system has been implemented, a post-implementation review is conducted to confirm that the transformation objectives and business benefits were achieved.

The scripts that were created in the previous steps are used here as a basis for integration testing as well as end-user training. Coordination between BPR, I.T., and now also Change Leadership (management) are 
paramount. During this phase, user testing ensures acceptance to the new system. Some things to watch for in this final phase are as follows:

Expect errors to surface in integration testing. Change management should be strict but not stifling.

\section{CONCLUSION}

There is nothing standard about ERP implementations as each effort is subject to a series of organizational-specific issues. This study provides a chronological look at three consecutive cases of SAP R/3 implementations that used the D\&T FastTrack $@$ for SAP implementation methodology. These three businesses, while distinctly varied in their nature, were wholly-owned by Bayer AG, a single multi-national manufacturing conglomerate. As such, the study is limited in nature to manufacturing and the nature of sister companies under the umbrella of a conglomerate. It is also limited to SAP R/3 as the ERP enabler software and to D\&T's FastTrack@ for SAP as the implementation methodology. Probably most importantly, however, is that the study was limited in terms of the financial information to which the active participants were privy.

Despite these limitations, this study might help companies, wishing to implement ERP systems, be more cognizant of potential sticking points. While the findings, as presented throughout this paper, are specific to a particular phase in the implementation process, there are five high-level themes that categorize the findings overall. These are as follows:

ERP is about Empowerment. Enabling employees at all levels to make better business decisions though information must remain the focus.

The process of becoming ERP enabled should evolve from the corporate culture; never should a corporation be stuffed into an ERP mold.

Corporations attempting ERP enablement must maintain realism. Avoiding over designing and over architecting is paramount in meeting deadlines.

Business decisions must drive the technological solutions and not vice versa.

Corporations must take the ownership for their ERP project from the consultants as soon as possible.

These themes form a basis for identifying the elements that Bayer AG changed in its implementation approach that may have contributed to its differentiated success with its ERP implementation endeavors. Corporations taking on the ERP challenge are never guaranteed success. However, being cognizant of these overlying themes throughout the process, as well as the more detailed recommendations throughout each step in the ERP implementation as described in this paper, could improve the odds of achieving a higher level of success.

\section{REFERENCES}

1. BOBA. (1998, Sept.). Best of Breed firms take on the ERP Providers. Retrieved September 8, 1998 from http://www.computronsoftware.com/ boba0998.htm

2. Basu, V., Lederer, A.L. (2004). An Agency Theory Model of ERP Implementation, ACM/SIGMIS Conference on Computer Personnel Research, 1, 8-13.

3. Beatty, R.C., Williams, C.D. (2006). ERP II: Best Practices for Successfully Implementing an ERP Upgrade, Communications of the ACM, 49, 105-109.

4. Besson, P., Rowe, F. (2001). ERP Project Dynamics and Enacted Dialogue Perceived Understanding, Perceived Leeway, and the Nature of Task-Related Conflicts, The DATA BASE for Advances in Information Systems, 32, 47-66. .

5. Boff, G.M. (2002). Implementing an Enterprise Resource Planning System: What Differentiates the Corporations that Make It from Those that Do Not, UMI Dissertation Publishing, ProQuest Information and Learning.

6. Deloitte \& Touche Consulting Group, "FastTrack for SAP." 1999, FastTrack Databases $^{\mathrm{TM}}$.

7. Nah, F.F., Zuckweiler, K.M., Lau, J.L. (2003). ERP Implementation Chief Information Officers' Perceptions of Critical Success Factors, Interaction Journal of Human-Computer Interaction, Vol.16, 5-22.

8. Shepard, J., Locke, B., D’Aquila, M. \& Carter, K. (2005). Market Analytix Report: The Enterprise Resource Planning Report, 20042009. AMR Research Report,.

9. Skovira, R. J. (2003). The Social Contract Revised: Obligation and Responsibility in the Information Society. Hershey, PA: Idea Group Publishing.

10. Soh, C., Sia, S.K., Tay-Yap, J. (2000). Cultural Fits and Misfits Is ERP a Universal Solution? Communications of the ACM, 43, 47-51.

11. Stake, R.E. (Ed.). (1995). The Art of Case Study Research (1st ed.). Thousand Oaks, CA: Sage Publications. 